einem Buch, in dem alle Bereiche der Holzbiologie in erschöpfender Weise sowie überaus klar und sprachlich vollendet dangeboten werden. Was alle Veröffentlichungen MAYER-WEgELINS auszeichnet, ist einerseits die enge Verbindung mit Wald- und Forstwirtschaft, andererseits wissenschaftliche Gründlichkeit und eine besondere Fähigkeit, dem Leser auch schwierige Zusammenhänge verständlich zu machen. Fern allem engen Spezialistentum hat er stets Fragestellungen aufgegriffen, die praktisch und wirtschaftlich von Bedeutung waren. Beobachtungen und Erfahrungen im Ausland fanden in sehr wertvollen Abhandlungen ihren Niederschlag, wie z. B. über Waldnutzung und Forstwirtschaft in der Türkei, über Aufforstungen in der Ukrainischen Steppe, oder die neueste Veröffentlichung - über forstlich-bodenkundliche Ermittlungen in einem nordanatolischen, von bäuerlichen Siedlungen durchsetzten Waldgebiet.

Gesprächsweise hat MAYER-WEGELIN einmal geäußert, daß er die unter seiner Leitung entstandenen Dissertationen als seine besten Arbeiten betrachte. Dies darf wohl so interpretiert werden, daß er als überaus erfolgreicher akademischer Lehrer sich besonders angelegentlich mit dem wissenschaftlichen Nachwuchs befaßt hat. Er verstand es, eine Anzahl begabter und interessierter Schüler um sich zu scharen und $\mathrm{zu}$ ausgezeichneten wissenschaftlichen Leistungen anzuregen, wie er überhaupt stets Arbeiten anderer Forscher großzügig und verständnisvoll gefördert hat. So entstand nicht nur in Hann.Münden und in Hamburg eine "Schule", die ganz von MAYERWegelins Geist und Persönlichkeit erfüllt war; es ergaben sich auch enge und herzliche Beziehungen $\mathrm{zu}$ in ihrer Arbeitsrichtung verwandten Forschungsinstituten des In- und Auslandes. Zahlreiche Schüler und Kollegen haben Professor MAYER-WEGELIN für vielerlei Rat, für seine ständige Hilfsbereitschaft und eine ideale Zusammenarbeit zu danken.

Die Emeritierung bedeutete für MAYER-WEgelin nicht eine Zeit des Ausruhens nach einer vielseitigen, angespannten Forschungs-, Lehr- und Verwaltungstätigkeit. Es folgten vielmehr Reisen, die ihn wieder, wie schon in früheren Jahren, in die Türkei und den Nahen Osten bis in das Zweistromland führten. Man darf sicher sein, daß die Ernte dieses reichen Forscherlebens noch keineswegs eingebracht ist und daß wir noch manche Ergebnisse und Anregungen von ihm erwarten dürfen.

Dem Jubilar seien die herzlichsten Glückwünsche zum 70. Geburtstag ausgesprochen; mögen ihm noch lange Jahre in voller Gesundheit, Spannkraft und Schaf: fensfreude beschieden sein!

von Pechmann

\title{
Tagung der Internationalen Akademie der Wissenschaft vom Holz
}

Die Internationale Akademie der Wissenschaft vom Holz, 1030 Wien, Arsenal Objekt 212, veranstaltet am 2. September 1967 im Wiener Saal des Mozarteums in Salzburg eine Tagung über Fragen der Grundlagenforschung dieses Fachgebietes.

Zur Vormittagssitzung, die vier wissenschaftliche Vorträge umfaßt, werden außer den Mitgliedern der Akademie, von denen schon Anmeldungen aus Australien, Japan, Indien, Nordamerika und verschiedenen europäischen Staaten vorliegen, auch die Mitglieder des "Vereins der Freunde und Förderer der International Academy of Wood Science" und an der einschlägigen Forschung interessierte Spitzen der Behörden und der Forst- und Holzwirtschaft eingeladen.

Am Nachmittag finden eine interne Akademiesitzung und ein gemeinsamer Empfang der Landesregierung des Bundeslandes und des Magistrats der Stadt Salzburg im Marmorsaal des Mirabellschlößchens statt. 